\title{
The Role of Cognitive Factors in the Development and Maintenance of Eating Disorders - the Concept of Thought Shape Fusion
}

\author{
An Interview with Jennifer S. Coelho
}

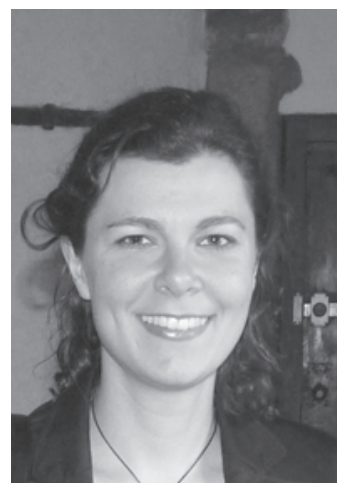

To increase the efficacy of currently applied treatment interventions in eating disorders (ED) the role of cognitive factors, such as consistent, non-veridical and skewed thinking, becomes increasingly evident.

Professor Jennifer Coelho is an important expert in the field of cognitive distortions in ED. Her research focuses on weight- and shape-related preoccupations such as the concept of Thought Shape Fusion (TSF) [Coelho et al., 2008]. This construct was developed according to the 'thought-action fusion' model in individuals with obsessive compulsive disorder (OCD) [Shafran et al., 1996]. OCD patients often assume that the mere thought of an action is morally equal to the real behavior and increases the probability of the feared event. As such, OCD patients believe that thinking of hurting someone is as morally wrong as actually committing the act or that thinking of being involved in a car crash might increase the probability of a real accident. The interview was conducted by Professor Simone Munsch.

How did theories about the role of cognitive factors in ED develop since the 2 factors theory of Connors [1996]?

Earlier models of ED, such as that put forth by Connors [1996], suggested that high levels of body dissatisfaction in combination with problems with self-regulation processes (e.g. affect regulation) increased vulnerability for ED. More recently, models of ED have evolved to include both cognitive and behavioral factors. For example, the transdiagnostic model of ED [Fairburn et al., 2003] suggests that ED symptoms develop as an interaction between cognitive factors (affect regulation, low self-esteem, over-evaluation of weight and shape, perfectionism), interpersonal factors, and behavioral factors (e.g. strict dieting). In the transdiagnostic model, over-evaluation of weight/shape represents one of the central cognitions in individuals with ED. Cognitive distortions in individuals with ED that are related to food/ weight/shape, such as TSF, represent a specific example of over-evaluation of weight/shape. By studying the processes that are thought to play a central role in $\mathrm{ED}$, we will be able to elucidate the cognitive factors involved in the maintenance of $\mathrm{ED}$, and in turn hopefully improve our current cognitive-behavioral treatments.

Professor Coelho, currently the concept of thought-action fusion of Shafran et al. [1996] has been adapted and transformed into the TSF model in ED patients. What are the characteristics of TSF in ED and how are patients thinking and feeling when experiencing TSF in daily life?

TSF, like thought-action fusion, occurs when individuals assume that mere thoughts increase the probability of feared events, and are morally equal to real behavior. TSF occurs specifically when thinking about eating high-caloric foods. People who experience TSF report believing that they have gained weight after they merely imagine eating high-caloric or 'fattening' foods, report feeling fatter after thinking about eating these foods, and also report feeling as though

\begin{tabular}{ll}
\hline KARGER & ๑ 2012 S. Karger GmbH, Freiburg \\
Fax +49 7614520714 & Accessible online at: \\
$\begin{array}{l}\text { Information@Karger.de } \\
\text { www.karger.com }\end{array}$ & www.karger.com/ver
\end{tabular}


they have done something morally wrong. Logically, just imagining eating high-caloric foods has no effects on actual body shape or size - actual food consumption (in the absence of sufficient caloric expenditure) is necessary for weight gain. According to Shafran et al. [1999], who initially proposed the concept, TSF may develop based on learning processes. Actually eating high-caloric foods can lead to feelings of fatness and fear of weight gain; therefore, if thinking about certain foods is regularly followed by eating this food, eventually the thoughts alone can trigger similar reactions to eating.

In our studies on TSF, we have asked women to imagine eating high-caloric foods, in order to induce TSF and study its effects. Some women with ED have had very strong reactions after imagining eating foods that they consider to be forbidden. For example, one participant with bulimia nervosa imagined eating a large serving of paella, and subsequently reported very strong anxiety and urges to binge, indicating to the experimenter that having a binge/purge episode was the only way to rid herself of these food-related thoughts. This example fits with our overall findings that women with ED report much higher TSF after imagining eating high-caloric foods, relative to imagining a neutral situation [e.g. Coelho et al., 2008], and suggests that TSF-related thoughts may be linked with some eating-disordered symptomatology. That being said, not all individuals with ED report experiencing $\mathrm{TSF}$, and some have little or no anxiety or fear of weight gain after imagining eating high-caloric foods. We are currently developing some projects to investigate what factors may increase susceptibility to TSF.

Which stimuli trigger TSF (nutrition, body image-related stimuli, others?)

TSF is thought to be triggered mainly by thoughts about eating fattening or high-caloric foods. The majority of experimental research on TSF has employed thoughts of food consumption to induce TSF [e.g. Radomsky et al., 2002; Coelho et al., 2008]. It seems that people need to be actively thinking about consuming food to experience TSF - mere exposure to high-caloric foods (such as a tray filled with chocolates, chips, and candy sitting beside participants) does not induce this phenomenon [Coelho et al., 2010]. However, some of our recent research has demonstrated that viewing salient television advertisements for high-caloric foods can induce some degree of TSF. We had a group of young adults watch either an advertisement for a McDonald's Big Mac or a neutral commercial for a telecommunications company. The participants who viewed the McDonald's advertisement reported significantly more TSF than did those who saw the neutral commercial [Werle and Coelho, 2012].

However, it appears that food-related stimuli are not alone in triggering the phenomenon of TSF. The questionnaire developed by Shafran and colleagues [1999] to assess TSF also includes questions about body image-related stimuli (such as thoughts about not exercising and thoughts about gaining weight), in addition to food-related stimuli. Therefore, it is possible that this phenomenon generalizes to other types of triggers. In support of this idea, Simone Munsch, Gregoire Zimmerman and colleagues have collected some pilot data indicating that exposure to thin ideals in the media may trigger some degree of TSF. More research is needed to tease apart the specific triggers for TSF, and compare and contrast TSF and general body dissatisfaction.

TSF in ED was adapted from OCD research. The 2 disorders share the tendency of obsessive behaviors and thoughts. Do you think an adapted TSF style could be present in other mental disorders such as depression or anxiety disorders?

Indeed, both ED and OCD share similar types of cognitions, and some have suggested that there may be a similar underlying etiology between these disorders. Similarly, individuals with ED exhibit high levels of thought-action fusion (to the same extent as do individuals with OCD). For this reason, we expected that there might be some shared vulnerability to TSF in individuals with OCD and ED. However, our recent research demonstrated that, in fact, individuals with OCD were much less susceptible to TSF than were individuals with ED, and exhibited lower tendencies towards TSF than did healthy women with no history of either an ED or OCD [Coelho et al., 2012a]. The presence of an adapted TSF style in individuals with other mental disorders, such as depression or anxiety, remains to be examined. However, our recent research on TSF in individuals with OCD provides some preliminary evidence that TSF is somewhat specific to eating pathology. In contrast, individuals with panic and generalized anxiety disorder are vulnerable to thoughtaction fusion [e.g. Rassin et al., 2001], and thought-action fusion may be mediated by depression [Abramowitz et al., 2003]. In this sense, some degree of cognitive distortions about the links between thoughts and action appear to be present in general psychopathology, including depression and anxiety disorders.

\section{Which state or trait factors might be associated with TSF susceptibility?}

Although individuals with ED are particularly susceptible to TSF, not every individual with an ED reports TSF after thinking about eating high-caloric foods. Yet, some healthy young women who have never had an ED exhibit some susceptibility to TSF [Coelho et al., 2008, 2010], which suggests that it isn't only those with ED who experience this phenomenon. One of the next steps in this area of research is to try to tease apart what factors are associated with TSF susceptibility. Recent research that we have collected examined which psychological traits might predict the presence of TSF. We demonstrated 
that numerous trait factors were predictive of TSF, including eating pathology, body dissatisfaction, depression, and thought-action fusion. The evidence for state factors that increase susceptibility to TSF is less clear - we have ruled out anxiety as a trigger for TSF [Coelho et al., 2008], but are currently investigating the impact of other state factors, such as body dissatisfaction.

Are there differences between TSF in different subgroups of ED patients, such as anorexia nervosa (AN), bulimia nervosa $(B N)$, and binge eating disorder (BED) or obesity?

The research on TSF to date has included either a transdiagnostic sample (grouping together patients with AN, BN, and ED not otherwise specified (EDNOS)) or has studied only one subtype of ED, e.g. AN [Radomsky et al., 2002]. It is therefore not yet possible to establish whether there are differences in TSF between patients with AN versus BN. According to the transdiagnostic model (and the DSM-IV diagnostic criteria for ED), all individuals with ED express an over-evaluation of weight/shape. TSF could be considered to be a specific example of the process of over-evaluation of weight/shape; therefore, we would not expect specific differences in TSF across ED subtypes. We are currently in the processes of investigating this question empirically. The presence of TSF in individuals with BED has not yet been investigated; however, some recent research suggests that individuals who are overweight or obese are less susceptible to this distortion than are individuals within the normal-weight range. A group of overweight women were less susceptible to a TSF induction than were normalweight women [Coelho et al., 2012b]. Similarly, a group of obese adolescents who were receiving in-patient weightloss treatment reported less susceptibility to TSF than did adolescents who were in the normal-weight range (Coelho, Siggen, Dietre and Bouvard, submitted). The study on adolescents also demonstrated that eating pathology (as measured by the EAT-26), but not obesity, was predictive of TSF.

Based on our examination of the literature on food-cue exposure, a pattern appears to be emerging in which overweight individuals respond to food-related cues with increased wanting for the food, in the absence of cognitions that may motivate reduced consumption of high-caloric foods. In line with this, researchers have proposed that individuals who are successful in losing/maintain weight successfully self-regulate after exposure to food temptations, whereas those who are overweight appear to have difficulties in self-regulating and following dieting goals [Ouwehand and Papies, 2010]. The lack of response of overweight individuals to the TSF induction supports this proposition. Overall, it appears that TSF is a phenomenon which is more relevant to individuals with ED, and individuals who are within the normal-weight range, than to individuals who are overweight.
Usually research in ED such as $A N$ and $B N$ relies solely on women, which seems justified as most patients are female. In $B E D$, on the contrary, a substantial part of the population is male. Do you expect gender-dependent cognitive mechanisms?

Indeed, the majority of research on cognitive factors in eating pathology (such as TSF) has included only women as participants. However, our recent work on susceptibility to TSF after exposure to television advertisements [Werle and Coelho, 2012; see question 2 for details] also included male participants. We demonstrated that only women were susceptible to TSF, reporting higher state TSF after watching a food advertisement than did males. It therefore appears that there may be some gender-dependent cognitive mechanisms. It may be that women are much more susceptible to TSF than are males because of their drive to lose weight. About half of the males who highly value weight and shape report being driven to gain weight, specifically muscle mass. Similarly, males are more likely to use exercise as a weight control technique, whereas women are more likely to restrict their intake [Drewnowski and Yee, 1987]. Future research that examines specific triggers for TSF, including thoughts about exercising or body dissatisfaction, may help elucidate the role of genderdependent mechanisms and identify circumstances under which males may be more susceptible to weight-/shaperelated cognitive distortions.

Which interventions do you imagine could be developed in order to target dysfunctional cognitive mechanisms such as TSF in ED?

One of the empirical questions that remain to be answered is whether we need to develop specific interventions to target dysfunctional cognitive mechanisms (such as TSF), or whether these distortions decrease naturally with cognitive-behavioral treatment for ED. Manualized cognitive-behavioral treatment for $\mathrm{BN}$ currently includes cognitive restructuring to address concerns about shape and weight. However, the guidelines for this process are very general. Therapists are instructed to choose a 'typical example' of a problematic thought [Fairburn et al., 1993, p. 385]. If specific cognitive distortions are present in a subset of patients who do not respond well to treatment, the addition of a treatment module specifically addressing these cognitions may be warranted. This module could potentially involve psychoeducation about specific relevant cognitions and distortions, followed by a focus on testing and restructuring these cognitions.

How could early detection of vulnerable individuals be accomplished?

A difficult question, which many researchers in the area of ED prevention have been working on! For example, prevention programs are sometimes offered universally (with the 
aim of reducing risk factors for ED in a large group of individuals), whereas some are targeted specifically to individuals with several identified risk factors (such as young women with high levels of body dissatisfaction or a strong internalization of the thin ideal). A recent meta-analytic review [Stice et al., 2007] demonstrated that prevention programs that were targeted towards specific vulnerable groups had larger effects than did universal prevention programs. Therefore, early detection of vulnerable individuals appears to be important. School-wide assessment and intervention programs could represent a promising avenue.

Alternatively - focusing on treatment as opposed to prevention - there is a movement to provide patient-centered care during treatment, and to match treatments to patients' profiles (which could include both genetic and psychological characteristics). Assessment of relevant cognitive factors, such as susceptibility to cognitive distortions, during evaluations could be envisioned to help in selecting relevant treatment modules for individual patients. Given that a 'gold standard' treatment for AN has not yet been identified, however, we have our work cut out for us to improve knowledge of treatment efficacy in the field of ED!

Dear Professor Coelho, thank you very much for this interview.

Thank you, Professor Munsch, for your invitation to participate in this special issue - I am pleased to have had the opportunity to discuss some of the state-of-the-art research into cognitive factors implicated in the etiology of ED.

\section{References}

Abramowitz JS, Whiteside S, Lynam D, Kalsy S: Is

thought-action fusion specific to obsessive-compulsive disorder? A mediating role of negative affect. Behav Res Ther 2003;41:1069-1079.

Coelho JS, Carter JC, McFarlane T, Polivy J: 'Just

looking at food makes me gain weight': experimental induction of thought-shape fusion in eatingdisordered and non-eating-disordered women. Behav Res Ther 2008;46:219-228.

Coelho JS, Roefs A, Jansen A: The role of food-cue

-xposure and negative affect in the experience of thought-shape fusion. J Behav Ther Exp Psychiatry 2010;41:409-417.

Coelho JS, Jansen A, Bouvard M: Cognitive distor-

tions in normal-weight and overweight women: susceptibility to thought-shape fusion. Cogn Ther Res 2012a:36:417-425.

Coelho JS, Baeyens C, Purdon C, Pitet A, Bouvard M:

Cognitive distortions and eating pathology: specificity of thought-shape fusion. Behav Res Ther 2012b;50:449-456.
Connors ME: Developmental vulnerabilities for eating disorders; in Smolak L, Levine M, Striegel-Moore R (eds): The Developmental Psychopathology of Eating Disorders. Hillsdale, NJ, Erlbaum, 1996, pp 285-310.

Drewnowski A, Yee DK: Men and body image: are males satisfied with their body weight? Psychosom Med 1987;49:626-634.

Fairburn CG, Marcus MD, Wilson GT: Cognitivebehavioral therapy for binge eating and bulimia nervosa: a comprehensive treatment manual; in Fairburn CG, Wilson GT (eds): Binge Eating: Nature, Assessment, and Treatment. New York, Guilford Press, 1993.

Fairburn CG, Cooper Z, Shafran R: Cognitive-behavior therapy for eating disorders: a 'transdiagnostic' theory and treatment. Behav Res Ther 2003;49:501528.

Ouwehand C, Papies EK: Eat it or beat it. The differ-

ential effects of food temptations on overweight and normal-weight restrained eaters. Appetite 2010:55:56-60.
Radomsky AS, de Silva P, Todd G, Treasure J, Murphy T: Thought-shape fusion in anorexia nervosa: an experimental investigation. Behav Res Ther 2002;40:1169-1177.

Rassin E, Diepstraten P, Merckelbach H, Muris P: Thought-action fusion and thought suppression in obsessive compulsive disorder. Behav Res Ther 2001;39:757-764.

Shafran R, Thordarson DS, Rachman S: Thought-

action fusion in obsessive compulsive disorder. J Anx Dis 1996;10:379-391.

Shafran R, Teachman BA, Kerry S, Rachman S: A

cognitive distortion associated with eating disorders: thought-shape fusion. Br J Clin Psychol 1999; 38:167-179.

Stice E, Shaw H, Marti CN: A meta-analytic review of

eating disorder prevention programs: encouraging findings. Annu Rev Clin Psychol 2007;3:207-231.

Werle COC, Coelho JS: Ironic effects of food commercials on cognitions and intentions. Poster presentation. Society for Consumer Psychology Winter Conference, Las Vegas, 2012. 